\title{
The interplay between multiple enhancer and silencer elements defines the pattern of decapentaplegic expression
}

\author{
Jian-Dong Huang, Deborah H. Schwyter, Jill M. Shirokawa, and Albert J. Courey ${ }^{1}$ \\ Department of Chemistry and Biochemistry, University of California, Los Angeles, California 90024-1569 USA
}

The product of the zygotically active decapentaplegic (dpp) gene appears to function as a morphogen that specifies positional information in the dorsal half of the Drosophila embryo. The dorsal-specific transcription of $d p p$ is the key step in establishing a morphogen gradient. We demonstrate here that multiple regions within the second intron of the gene cooperate with one another to generate the wild-type level and pattern of $d p p$ transcription. These regions contain both generalized enhancer elements as well as ventral-specific repressor elements. Placed within the context of heterologous promoters, the intron retains its ability to direct general activation and ventral repression. The ventral specific repression of $d p p$ transcription is directly mediated by binding sites for the dorsal ( $d l)$ morphogen in the repressor elements. In contrast with the zerknüllt (zen) ventral repressor element, which contains a few high-affinity $d l$-binding sites, $d p p$ contains multiple relatively low-affinity sites that function together to bring about complete ventral repression. Because $d p p$ and zen have nearly coincident early expression domains, these results indicate that the same boundary of repression can be specified by $d l$-binding sites of different affinity. We discuss the possibility that unknown factors interact with $\boldsymbol{d l}$ protein to determine the domain of $\boldsymbol{d l}$-mediated repression.

[Key Words: decapentaplegic; transcription; Drosophila embryogenesis; transcriptional silencer; dorsal morphogen; DNA-binding protein]

Received December 17, 1992; revised version accepted January 26, 1993.

decapentaplegic $(d p p)$ is a zygotically active gene that plays a pivotal role in the specification of positional information in the early Drosophila embryo (Irish and Gelbart 1987; Ferguson and Anderson 1992). The $d p p$ protein may be distributed in a dorsal-to-ventral gradient and has the properties of a morphogen, because it is able to specify multiple cell fates in a concentration-dependent manner (Ferguson and Anderson 1992). The spatially localized transcription of $d p p$ is the critical initial event required to establish the $d p p$ morphogen gradient. In the early syncytial blastoderm, $d p p$ is transcribed in the dorsal $40 \%$ of the embryo /the anlagen of the dorsal ectoderm and the amnioserosa; St. Johnston and Gelbart 1987; Ray et al. 19911. Translation of the $d p p$ message results in the synthesis of a secreted protein with homology to the TGF- $\beta$ family of growth factors (Padgett et al. 1987). Diffusion of this protein from its region of synthesis may then result in the establishment of an activity gradient.

The spatially restricted transcription of $d p p$ in the blastoderm is the result of an earlier morphogen gradient, which is established by the action of a large set of maternally active genes (Anderson 1989). These genes act during oogenesis to set up signaling pathways between the soma and the germ line, which ultimately

${ }^{1}$ Corresponding author. result in the spatially regulated nuclear uptake of the dorsal (dl) gene product in the early embryo (Roth et al. 1989; Rushlow et al. 1989; Steward 1989). As a consequence of this spatially regulated nuclear translocation, the nuclear concentration of $d l$ protein is graded along the dorsal-ventral axis of the blastoderm embryo, with highest concentrations in the ventral-most nuclei and lowest concentrations in the dorsal-most nuclei.

The $d l$ protein is a sequence-specific DNA-binding transcription factor that regulates the expression of a number of zygotically active genes in a concentrationdependent manner. High concentrations of $d l$ result in the expression of genes normally active in the ventrally located mesodermal anlagen, such as twist and snail (Thisse et al. 1987; Jiang et al. 1991; Pan et al. 1991; Thisse et al. 1991; Ip et al. 1992a). Intermediate concentrations of $d l$ allow the expression of genes that are normally transcribed in ventrolateral regions, such as rhomboid and single-minded (Ip et al. 1992b; Kasai et al. 1992). Finally, low concentrations of $d l$ (or no $d l$ ) result in the expression of genes that are normally active in dorsal and dorsolateral regions, such as $d p p$, zerknüllt (zen), and tolloid (Rushlow et al. 1987; St. Johnston and Gelbart 1987; Shimell et al. 1991).

In embryos that lack the $d l$ gene product, $d p p$ is transcribed uniformly throughout the blastoderm (Ray et al. 1991\}. This suggests that the $d p p$ gene contains one or more generalized enhancer elements that, in the absence 
of the $d l$ transcription factor, serve to activate $d p p$ throughout the embryo. In addition, it suggests that $d l$ can interact with the $d p p$ gene to overcome the activating effects of these generalized enhancers. A precedent for this type of regulation comes from studies of the zen gene, the initial expression pattern of which is nearly identical to that of $d p p$ (Ray et al. 1991). zen has been shown to contain a single silencer element that can interact with $d l$ to block the activity of a wide variety of heterologous enhancers (Doyle et al. 1989; Ip et al. 1991; Jiang et al. 1992). This silencer, termed a ventral repressor element (VRE), contains a few high-affinity $d l$-binding sites that are essential for its activity.

In the experiments presented here, we show that the $d p p$ second intron contains multiple general enhancer elements as well as multiple $d l$-dependent VREs. The pattern and level of $d p p$ expression in the early embryo is the result of the interplay of these numerous elements. In contrast to the zen VRE, which contains a few highaffinity $d l$-binding sites, the $d p p$ VREs contain numerous weak $d 1$-binding sites, at least some of which are critical for function. These results indicate that the domain of transcriptional repression mediated by $d l$ protein is not a simple function of the in vitro affinity of the target gene for $d l$ protein.

\section{Results}

The dpp second intron is necessary and sufficient to direct dorsal-specific transcription in the blastoderm embryo

Previous studies have indicated that the embryonic ex- pression of $d p p$ can be divided into several phases (St. Johnston and Gelbart 1987; Ray et al. 1991). In the blastoderm embryo, $d p p$ transcripts are first detected in the dorsal $40 \%$ of the embryo (the anlagen of the amnioserosa and the dorsal ectoderm), as well as in the anterior and posterior poles of the embryo (Fig. 1A). Later, during germ-band elongation, expression is lost at the dorsal midline and becomes restricted to broad lateral domains on both sides of the embryo (Fig. 1B). These broad domains correspond to the anlagen of the dorsal ectoderm. As germ-band extension nears completion, this pattern is replaced by four narrow longitudinal stripes, two on either side of the embryo, that exhibit segmental modulation (Fig. 1C).

Genetic analysis of the $d p p$ locus indicates that sequences extending from $\sim 1 \mathrm{~kb}$ upstream of the major early embryonic start site to just downstream of the coding region are sufficient for normal embryogenesis (St. Johnston et al. 1990). P-element-mediated germ-line transformation studies, however, reveal that the $5^{\prime}$ flanking region of the $d p p$ gene is not sufficient to drive dorsal-specific expression of a lacZ reporter gene in the blastoderm embryo (Fig. 1E). This region of DNA does, however, direct much of the $d p p$ expression seen during the later stages of embryogenesis (Fig. 1F,G; R. Padgett, pers. comm.).

The above result indicates that the sequences directing the expression of $d p p$ in the blastoderm embryo are not found within the $5^{\prime}$-flanking region and must therefore lie somewhere downstream of the transcriptional start site. Because sequences downstream of the $d p p$ coding region are not required for embryonic develop-
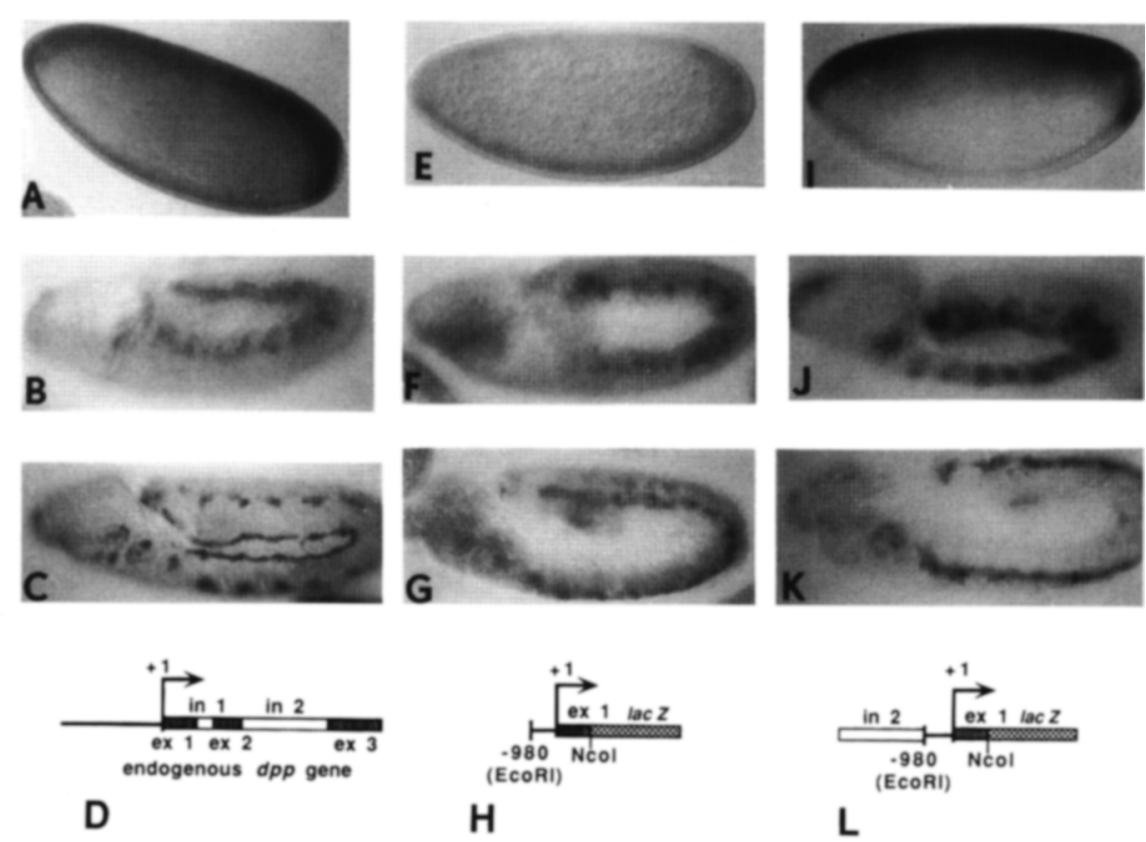

multiple independently transformed lines|, we cannot rule out the possibility that it results from elements present in the P-element vector sequences. The dorsal-lateral stripe seen for the endogenous $d p p$ gene in stage 12 embryos $(C)$ is missing or very weak in the patterns directed by the lacZ fusion constructs $(G, K)$. The segmental modulation of the ventral-lateral stripe is not as extreme for the lacZ fusions as it is for the endogenous gene.

Figure 1. Expression patterns of the endogenous $d p p$ gene and $d p p-l a c Z$ transgenes at different developmental stages. Expression patterns of $d p p$ mRNA in $w^{1118}$ embryos $(A-C)$ or lacZ mRNA in transgenic embryos $(E-G, I-K)$ were detected by whole-mount in situ hybridization with digoxigenin-labeled probes. The embryos shown in $E-G$ bear the P-element construct $-980 / d p p$ (shown in $H$ ). The embryos shown in $I-K$ bear the P-element construct $1-7^{\prime}-980 / d p p$ (shown in $L$ ). Embryos, which are viewed in sagittal section $(A, E, I)$ or on the surface $(B, C, F, G, J, K)$, are oriented so that dorsal is up and anterior is to the left. In blastoderm embryos, the $1-7^{\prime}-980 / d p p$ construct directs dorsal-specific expression $\langle I\rangle$, whereas the $-980 / d p p$ construct does not $\{E\}$. In stage 9 embryos, both constructs direct near wild-type expression patterns $(F, \eta)$. Note the abnormal anterior expression in $F$ and $I$. Although this ectopic expression is not a position effect (i.e., it is observed with 


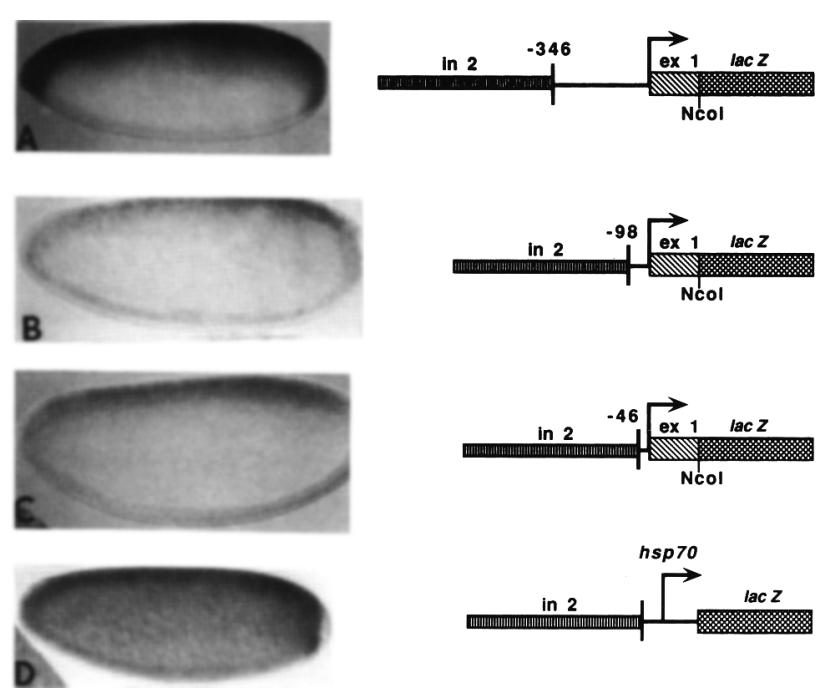

Figure 2. $d p p$ second intron is sufficient to direct dorsal-specific expression in Drosophila blastoderm embryos, lacZ mRNA distributions are revealed by in situ hybridization with digoxigenin-labeled probes. Embryos, which are oriented as in Fig. 1, are viewed in sagittal section. The expression patterns of $1-7^{\prime}-346 / d p p(A), 1-7^{\prime}-98 / d p p(B), 1-7^{\prime}-46 / d p p(C)$, and intron-hsp70/lacZ $(D)$ are dorsal specific.

ment, a likely place to look for regulatory elements is within one of the two introns that interrupt the coding region (Fig. 1D). The finding that the Drosophila virilis and Drosophila melanogaster dpp genes show significant homology within their second introns (R. Padgett, pers. comm.) prompted us to examine this portion of the $d p p$ locus for potential transcriptional control elements. $A \sim 2-k b$ genomic fragment containing the entire second intron was fused upstream of the $5^{\prime}$-flanking region of the $d p p$ promoter driving expression of the lacZ reporter (Fig. 1L). When introduced into embryos, this construct was found to direct strong dorsal-specific transcription of the reporter gene in the early blastoderm embryo (Fig. 1II. Thus, sequences within the second intron seem to be required for the dorsal-specific expression of $d p p$ in the blastoderm.

To determine whether the second intron is sufficient for spatially restricted expression, it was fused to a series of $5^{\prime}$ deletion variants of the $d p p 5^{\prime}$-flanking region. These variants have $5^{\prime}$ endpoints at $-582,-346,-98$, -46 , or -22 relative to the transcriptional start site. The second intron was found to direct dorsal-specific expression from each of these five deletion variants (representative data are shown in Fig. $2 \mathrm{~A}-\mathrm{Cl}$. In addition, the second intron was found to direct dorsal-specific expression from the $h s p 70$ core promoter element (Fig. 2D). Thus, sequences within the second intron appear to be both necessary and sufficient to direct dorsal-specific transcription from a core promoter element.

It is worth noting that although the $5^{\prime}$-flanking region of $d p p$ is dispensable for early dorsal-specific transcription, the region upstream of -98 contributes to the over- all level of this transcription. In particular, constructs lacking sequences upstream of -98 show consistently weaker expression patterns than the longer constructs (cf. Fig. 2A,B).

To demonstrate that the dorsal-specific expression driven by the second intron is dependent on the dorsal morphogen, the intron- $h s p 70$ construct (Fig. 2D) was crossed into a dl mutant background. Under these circumstances, uniform expression is observed along the entire dorsal-ventral axis (Fig. $3 \mathrm{C}$ ). When the transgenes were introduced into a Toll ${ }^{D}$ background, the opposite result was obtained. In these embryos, $d l$ protein is constitutively localized to the nucleus throughout the embryo (Roth et al. 1989; Steward 1989|. As a result, constructs bearing the second intron are repressed throughout Toll ${ }^{D}$ embryos with the exception of the anterior and posterior poles (Fig. 3B). Previous genetic analysis of $d p p$ regulation indicates that this terminal expression is driven by a separate maternal system and is independent of the $d l$ morphogen (Ray et al. 1991). Consequently, in addition to containing elements that direct dorsal-specific expression, the 5 -flanking region and/or second intron also contain terminal-specific transcription elements.

\section{The dpp second intron acts as a transcriptional silencer}

The above data strongly suggest that the second intron contains one or more elements that direct generalized transcriptional activation, together with $\mathrm{dl}$-dependent elements that overcome the action of these generalized activators on the ventral side of the embryo. An alterna-

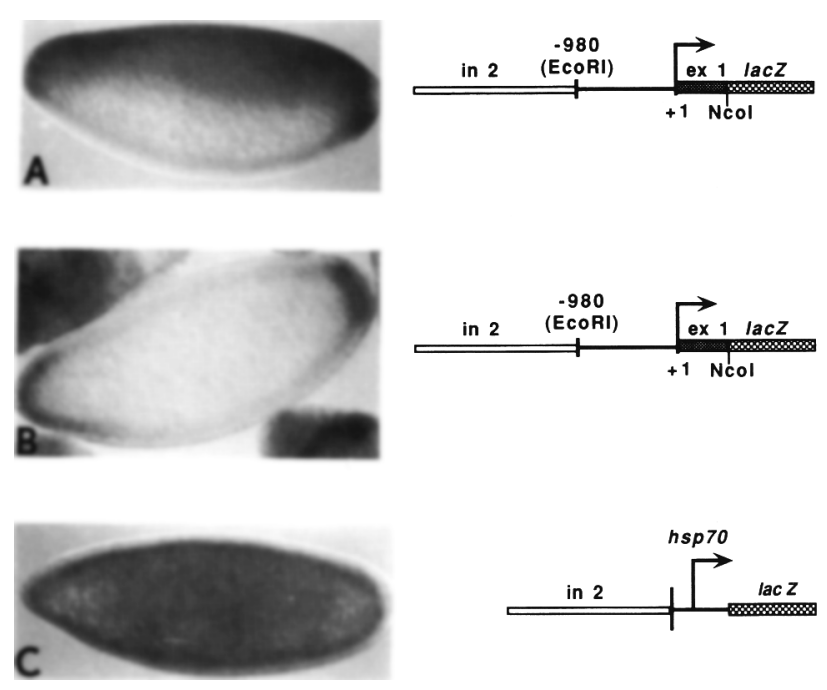

Figure 3. The dorsal-specific expression of the reporter gene is dependent on $\mathrm{dl}$ activity. Expression pattern of $2-7^{\prime}-980 / d p p$ was examined in wild-type $(A)$ and Toll gain-of-function $\left|T o l I^{10 b}\right|$ backgrounds $(B)$. The expression of intron-hsp $70 /$ lacZ was tested in a $d l$ loss-of-function $\left(d l^{1}\right)$ background $(C) .1 a c Z$ mRNA patterns were visualized by in situ hybridization using digoxigenin-labeled probes. Embryos are oriented as in Fig. 1. Blastoderm-stage embryos are displayed. 
tive explanation, however, is that spatially regulated expression of $d p p$ in the blastoderm is attributable solely to the action of an activator element that only functions dorsally. To distinguish between these two possibilities, we decided to determine whether the second intron could specifically silence a heterologous promoter on the ventral side of the embryo. For this experiment, we fused the $d p p$ second intron to a promoter that consists of a fragment of the Krüppel $(K r)$ gene driving transcription from the $h s p 70$ core promoter element. By itself, this $\mathrm{Kr} / \mathrm{hsp} 70$ promoter directs expression in a broad stripe that extends along the entire dorsal-ventral axis /Hoch et al. 1990; Fig. 4D,E). As has been shown for the zen ventral repressor (Ip et al. 1991), the addition of the $d p p$ second intron results in the ventral repression of this stripe. Specifically, the stripe is converted into a wedge that becomes both narrower and significantly weaker as it goes from the dorsal to the ventral side of the embryo (Fig. 4A,B). The gradually decreasing width of this wedge suggests that it results from a gradient of repressing activity. We conclude that the second intron contains one or more silencers that function as $d l$-dependent ventral repressor elements. Upon prolonged staining (Fig. 4B), we begin to see expression across the entire dorsal surface of the embryo. This is presumably the consequence of general enhancers present within the second intron.

\section{The dpp second intron contains multiple ventral repressor elements and generalized activator elements}

The results shown above establish that the second intron contains both a generalized enhancer activity as well as a ventral-specific silencing activity. To further delineate the elements responsible for these activities, we initiated a deletion analysis of the second intron using poly-
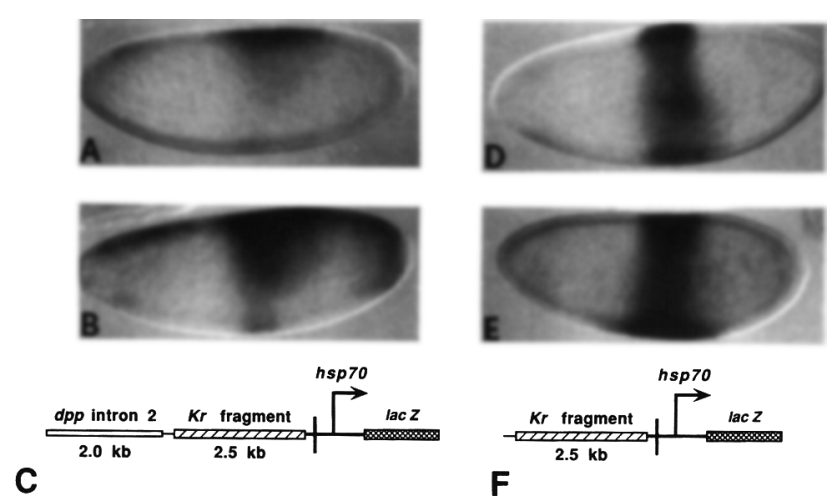

Figure 4. The $d p p$ second intron can silence a heterologous activator at a distance. lac $Z$ mRNA distributions are revealed by in situ hybridization with digoxigenin-labeled probes. Embryos, which are oriented as in Fig. 1, are viewed in sagittal section. The $K r-h s p 70 / l a c Z$ construct $(F)$ directs strong expression of the $l a c Z$ gene in a stripe that runs along the dorsalventral axis $(D$, lightly stained; $E$, heavily stained). The addition of the $d p p$ second intron in the $d p p$ intron-Kr-hsp70/lacZ construct $(C)$ results in the repression of this stripe on the ventral side of the embryo (A, lightly stained; $B$, heavily stained). merase chain reaction (PCR) amplification to generate various second intron subfragments. The results shown in Figure 5 indicate that the intron contains at least two regions that are capable of directing transcription that is specific for the dorsal side of the early embryo. When the intron was divided into three large, slightly overlapping fragments (Fig. 5G, fragments $1-2^{\prime}, 3-5^{\prime}$, and $6-7^{\prime}$ ), the $5^{\prime}$-fragment $\left(1-2^{\prime}\right)$ had no activity, the central fragment $\left(3-5^{\prime}\right)$ directed a strong dorsal-specific expression pattern (Fig. 5E), and the $3^{\prime}$-fragment $\left(6-7^{\prime}\right)$ directed a weak dorsal-specific pattern (Fig. 5D). More conclusively, the nonoverlapping $1-4^{\prime}$ and 6-7' regions both direct expression that is largely specific for the dorsal side of the embryo (Fig. 5D,E). Both regions must therefore contain a functional generalized activator element as well as a ventral repressor element. The multiple ventral repressor elements may work cooperatively, because ventral repressor elements present in the $1-4^{\prime}$ (Fig. 5F) and 4-7' (Fig. 5C) fragments each give only partial repression on the ventral side of the embryo, whereas the combination results in complete ventral repression (Figs. 1 I and 5A,B).

The dorsal-specific expression pattern seen with the 6-7' fragment (i.e., the fragment from the 3 ' end of the intron; Fig. 5D/ is rather weak at the syncytial blastoderm stage, and is not easily visible until cellularization is complete. We believe, however, that the ventral repressor element within this fragment is functional in the syncytial blastoderm, because deletion of this repressor from the 4-7' fragment results in complete derepression on the ventral side of the early embryo (fragment 4-5', see Fig. 8A,C below).

The ventral repressor and generalized activator elements in the $3-5^{\prime}$ region can function not only in the blastoderm stage embryo but also during germ-band elongation. Thus, if we attach a fully functional second intron fragment to the $d p p 5^{\prime}$-flanking region, we observe normal lateral expression in the germ-band-elongated embryo (Fig. 6B). A deletion of the intron that gives weak expression on the ventral side of the blastoderm embryo, however, also results in ventral expression in the germ-band-elongated embryo (Fig. 6C). This is surprising given that in the complete absence of any second intron sequences, the $d p p 5^{\prime}$-flanking region directs the expression of normal lateral stripes at this stage (Fig. 1F). Thus, there are apparently redundant mechanisms for preventing expression of the $d p p$ gene in ventral regions during germ-band elongation.

\section{The ventral repression of dpp is mediated by weak dl-binding sites}

To guide our further dissection of the intron, we systematically mapped the $d l$-binding sites in the entire intron. Using the $\mathrm{SAAB}$ technique, the consensus-binding site for the $d 1$ protein was found to be GGG(T/A) $\left.\right|_{4-5} \mathrm{CC}$ (Pan and Courey 1992). The outer Gs and Cs are especially critical for high-affinity binding, and, in the center of the sequence, there is a clear preference for Ts over As on the strand with three Gs. Because we expected the $d p p$ VRE to contain high-affinity $d l$-binding sites (see Discussion), 


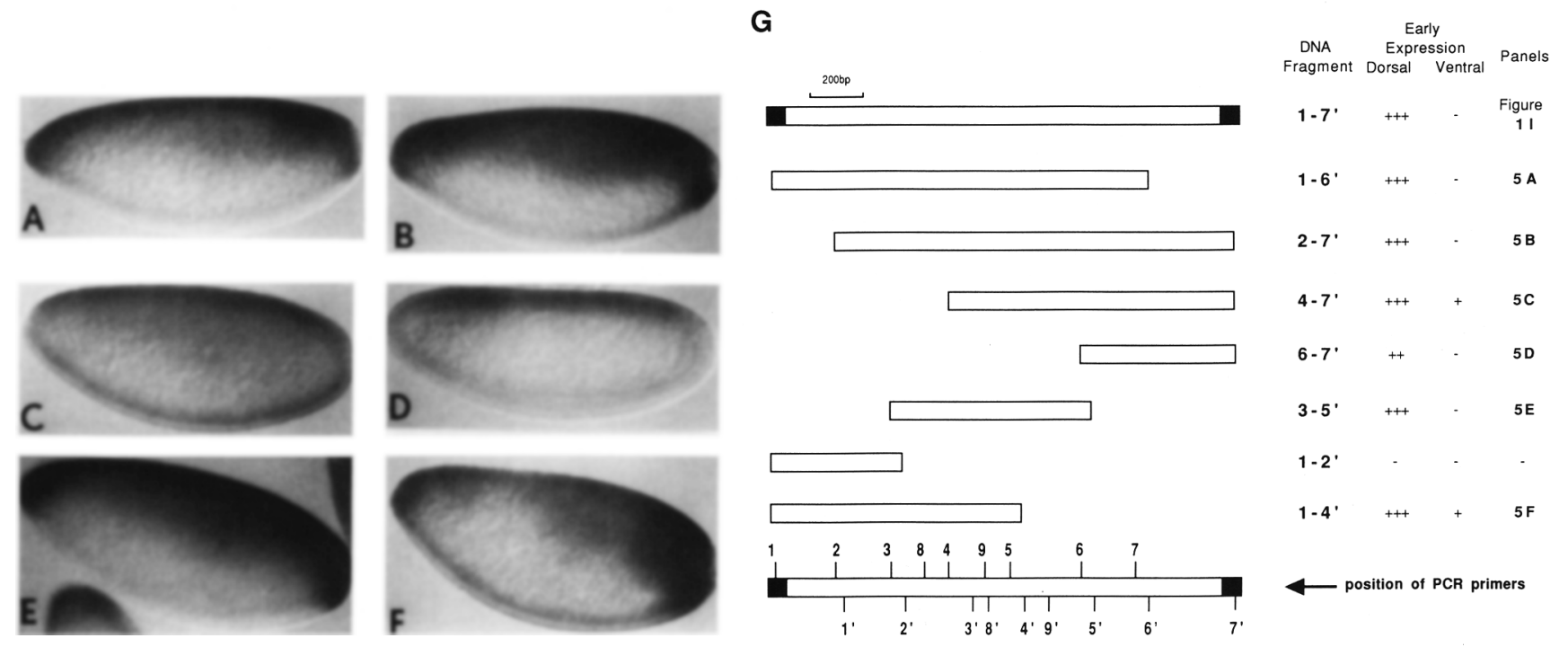

Figure 5. Dissection of the $d p p$ second intron reveals multiple general activator and ventral-specific repressor elements. The $I a c Z$ expression patterns directed by the 1-6'-980/dpp $(A), 2-7^{\prime}-980 / d p p(B), 4-7^{\prime}-980 / d p p(C), 6-7^{\prime}-980 / d p p(D), 3-5^{\prime}-980 / d p p(E)$, and $1-4^{\prime}-980 / d p p(F)$ transgenes were detected by in situ hybridization with a digoxigenin-labeled probe. Embryos, which are oriented as in Fig. 1, are viewed in sagittal section. Blastoderm-stage embryos are displayed with the exception of the $6-7^{\prime}-980 / d p p$ embryo $(D)$, which is just beginning gastrulation. $(G)$ Summary of constructs showing their approximate relative levels of expression on the dorsal and ventral sides of the blastoderm embryo.

we were surprised when a computer search of the second intron failed to turn up any consensus sites. When we allowed for one mismatch with the consensus, four sites were found (Fig. 7C). In each case, however, the mismatch was in one of the critical Gs or Cs.

To determine whether the intron contained sites that did not match the consensus, we used DNase I footprinting assays to search the entire region for $d l$-binding sites. These assays (representative examples of which are shown in Fig. 7A) utilized a near homogeneous preparation of baculovirus-expressed $d l$ protein. This search revealed a number of $d l$-binding sites in the second intron that were missed by the computer search. In Figure 7B, we have grouped these sites into two classes. S sites required $\sim 10 \mathrm{ng}$ of our baculoviral $d l$ preparation for good protection, whereas $M$ sites required $\sim 30 \mathrm{ng}$ of $d I$ protein for good protection. Additional sites (W sites) were found when higher concentrations of protein were used in the assays (these are not shown in Fig. 7B,C). An analysis of the protected sequences shows that all of the sites exhibit some homology to the previously defined consensus. Most of the elements, however, contain only a partial site (i.e., a stretch of two or three Gs followed by a run of several As and Ts). Given the low quality of the matches with the consensus, it is not surprising that electrophoretic mobility retardation assays show that even the strongest sites in $d p p$ are about 10 -fold weaker than the high-affinity sites found in the zen VRE (fig. 7D, E). Three of the four sites found by the computer search were also found in the DNase I footprint assays (sites S3, S4, and S8). The fourth site identified by the computer (aGGTAATCC) was never observed to bind $d l$ protein. The reason for this is unclear, although the context of a
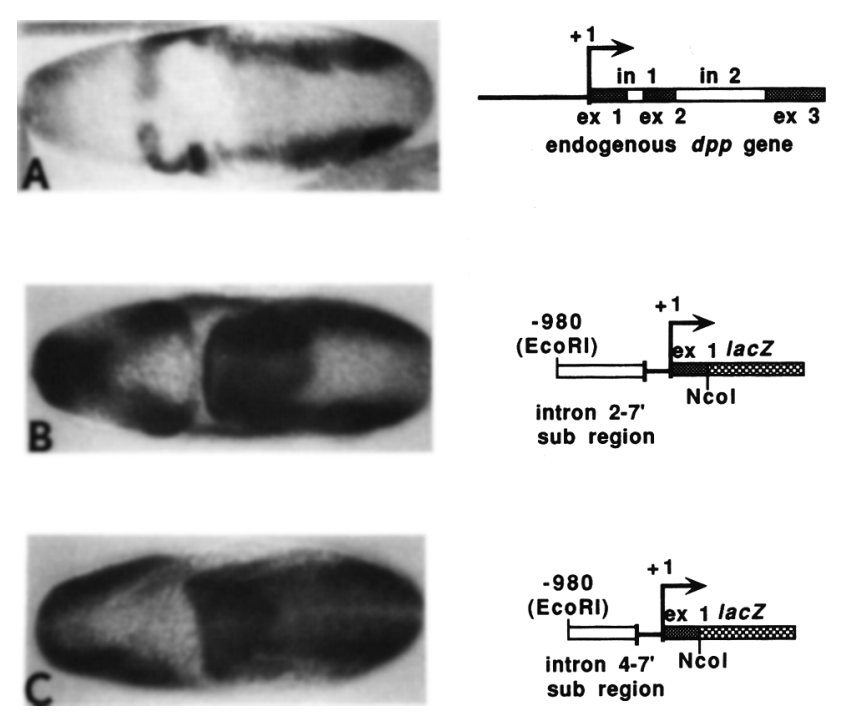

Figure 6. Ventral-specific repressor elements in second intron are functional in germ-band-elongated embryos. Endogenous $d p p(A)$ or $\operatorname{lac} Z(B, C)$ expression patterns were detected by in situ hybridization. Embryos are oriented with their dorsal sides facing the reader; anterior is to the left. Germ-band-elongated embryos are displayed; whereby the posterior ventral portion of the embryos can be seen in these dorsal view pictures. The wild-type $d p p$ expression pattern at this stage $(A)$ consists of two broad lateral stripes separated by a nonexpressing region along the ventral midline. Complete ventral repression of $l a c Z$ expression is observed in the $2-7^{\prime}-980 / d p p$ embryos $(B)$ but not in the $4-7^{\prime}-980 / d p p$ embryos $(C)$. Note the ectopic terminal (anterior and posterior) expression in the transgenic embryos $(B, C)$. 
A

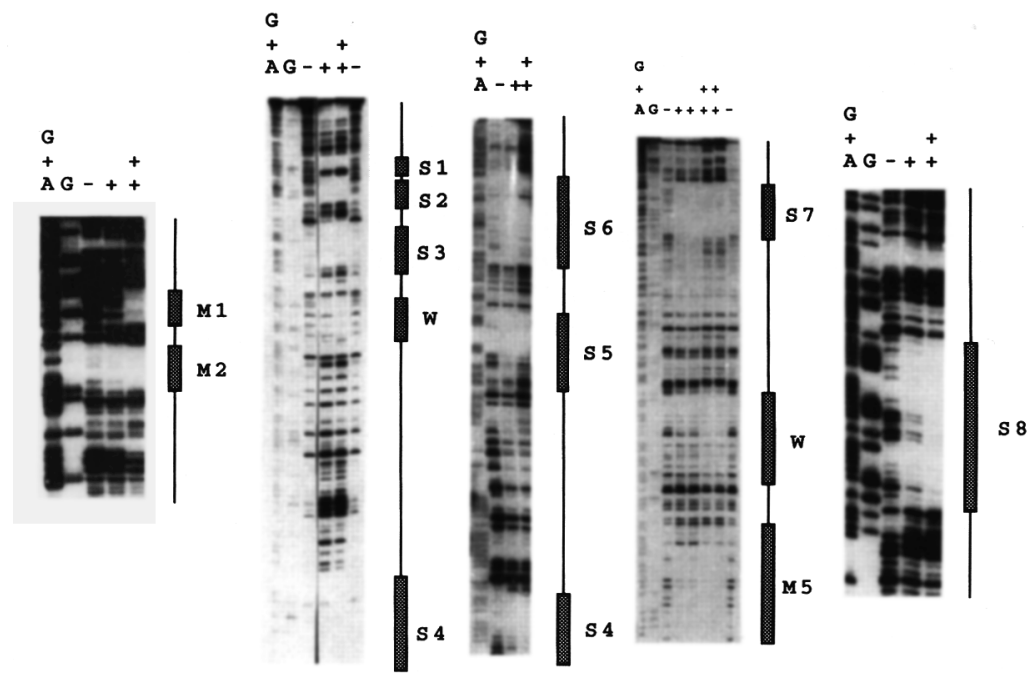

dl sites identified by computer search

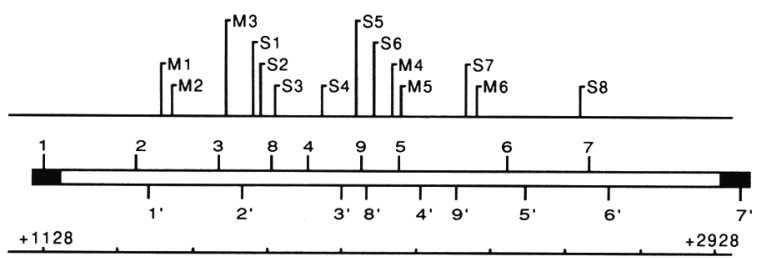

dl sites identified

by footprint assays

D

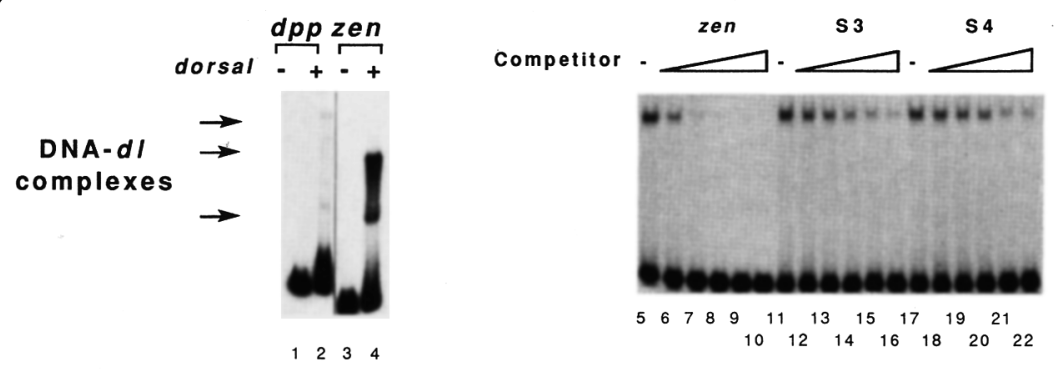

E

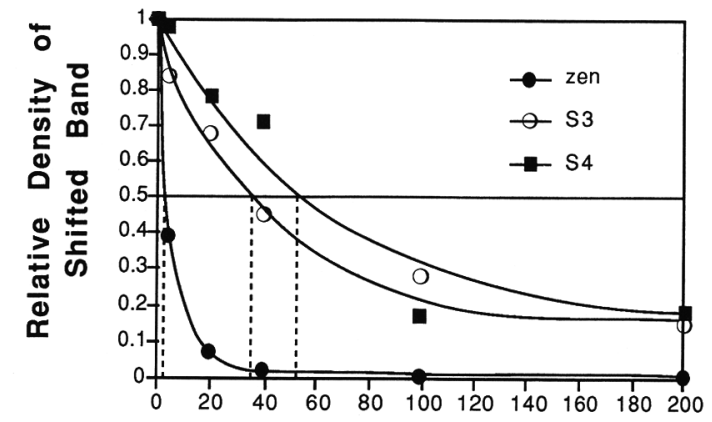

Cold Competitor (nM)

Figure 7. $d l$ protein interacts with the $d p p$ gene directly. (A) The five 5'-end-labeled probes shown here were generated using PCR-amplified intron fragments cloned into pBluescript. From left to right, the five probes used in the footprinting reactions are $2-2{ }^{\prime}, 3-3^{\prime}, 4-4^{\prime}, 5-5^{\prime}$, and $6-6^{\prime}$. Probes were incubated with no protein $(-1, \sim 10 \mathrm{ng}$ of pure baculovirus $d l$ protein $(+)$, or $\sim 30$ $\mathrm{ng}$ of dl protein $(++)$. A $+\mathrm{G}$ and $\mathrm{G}$ chemical sequencing ladders were included. Footprinted regions are represented by the shaded boxes. ( $B)$ Alignment of dl-binding sites with the consensus sequence. Each binding site is labeled with either an S followed by a number or an $\mathrm{M}$ followed by a number (see text for significance of this nomenclature) and corresponds to a shaded box in part $A$. The two possible consensus sites (Pan and Courey 1992) are labeled C (W signifies A or $T$ ). Uppercase letters indicate matches with the consensus. $(C)$ Positions of $d l$-binding sites identified by computer search and DNase I footprint assays. The position numbers shown on the scale at the bottom are relative to the $5^{\prime}$ end of the $d p p$ transcript $\mathbf{A}$ /St. Johnston et al. 1990). The S3, S4, and S8 sites were identified by both searches. $(D)$ Comparison of $d l$-binding sites in $d p p$ with high affinity sites in zen. Mobility retardation assays were performed as described by Pan et al (1992). The $d p p$ probe (lanes 1,2$)$ is the 3-3' PCR fragment from the second intron containing sites $\mathrm{M} 3, \mathrm{~S} 1, \mathrm{~S} 2, \mathrm{~S} 3$, and $\mathrm{S} 4$. The zen probe $\{$ lanes 3,4 ) is an $\mathrm{XbaI-NarI}$ fragment from zen that contains a single highaffinity site (the site at -1541 relative to the zen transcriptional start site; Ip et al. 1991). Lanes 5-22 represent 0.1 pmole of oligonucleotide probe containing a high-affinity consensus $d l$-binding site probe ( $P$ an and Courey 1992). Competitor oligonucleotides contained a high-affinity binding site from the zen VRE (the site at - 1217; Ip et al. 1991, lanes 6-10), the S3 site from $d p p$ (lanes 12-16), or the S4 site from dpp (lanes 18-22). Reactions contained 0.1 pmole (lanes 6,12,18), 0.5 pmole (lanes 7,13,19), 1 pmole (lanes $8,14,20$ ), 2.5 pmole (lanes $9,15,21$ ), or 5 pmole (lanes $10,16,22$ ) of the indicated competitors. Binding reactions contained no $d l$ protein (lanes 1,3 ) or $1 \mu l$ of bacterially synthesized dl-378 protein (lanes 2,4,5-22). (E) Quantitation of the data in $D$ (lanes 5-22). Band density was determined by densitometry and plotted as a function of the concentration of the various competitors. Broken lines extrapolated to the $\mathrm{x}$-axis indicate the amount of each competitor required to reduce the intensity of the shifted complex by a factor of 2 . (O) zen; $(O) \mathrm{S3}$; (a) S4. 
site is known to influence its binding affinity (Pan and Courey 1992).

Noting that most of the stronger sites are clustered in the 3-5' fragment, we decided to further dissect this region by P-element-mediated transformation. Deletion of the $5^{\prime}$ quarter of this fragment (construct 4-5'; Fig. 8A,C) results in the complete loss of ventral repression, such that the construct is expressed at high levels both dorsally and ventrally. Thus, the deleted region, which contains four $d l$-binding sites, must contain all or part of a ventral repressor element, whereas the $4-5^{\prime}$ construct itself must contain a generalized activator element. The deleted region contains four $d l$-binding sites. The $8-8^{\prime}$ construct, which overlaps the $5^{\prime}$ end of the 4-5' construct, allows us to further narrow down the location of these elements. This fragment, which contains three $d I$ binding sites, directs significantly stronger expression on the dorsal than on the ventral side of the embryo (Fig. $8 \mathrm{~B}, \mathrm{Cl}$. Thus, elements critical for ventral repression must lie in the 5' $100 \mathrm{bp}$ (the region between PCR primers 8 and 4), whereas elements critical for generalized activation most likely lie in the $3^{\prime} 170 \mathrm{bp}$ (the region between primers 4 and $8^{\prime}$ ) of this 270 -bp fragment.

To prove that the activity of the ventral repressor element in the $8-8^{\prime}$ fragment is mediated by $d l$-binding sites, point mutations were introduced into the two $5^{\prime}$ most dl-binding sites in this fragment /sites S3 and S4; i.e., the only two sites in this region identified by both the footprinting assays and the computer search). $\mathrm{Mu}-$ tagenesis of these two $d l$-binding sites completely eliminated the dorsal-ventral asymmetry in the expression pattern, resulting in uniform expression levels along the entire axis (Fig. 9, cf. $\mathrm{H}$ and J). When these same two dl-binding sites were mutated in the context of the longer 3-5' fragment, the dorsal-ventral asymmetry in the expression pattern was markedly reduced, although not completely eliminated. The wild-type $3-5^{\prime}$ fragment (Fig. 9B,C) drives an expression pattern that is completely specific for the dorsal side of the embryo and exhibits a sharp boundary between expressing and nonexpressing regions. In contrast, the mutated 3-5' fragment exhibits a graded expression pattern, and moderate levels of expression are detected all the way to the ventral midline (Fig. 9E,F). Thus, whereas the S3 and S4 sites play an important role in controlling the overall $d p p$ expression pattern, ventral repression seems to result from the additive effects of numerous $d l$-binding sites.

\section{Discussion}

We have shown that the second intron of $d p p$ contains multiple enhancers capable of mediating generalized transcriptional activation in the blastoderm, as well as multiple $d l$-dependent silencer elements that block the function of linked enhancers. In particular, a fragment from the center of the intron (the 3-5' fragment; Fig. $5 E, G$ ) contains elements that direct strong expression on the dorsal side of the early embryo, whereas a fragment from the $3^{\prime}$ end of the intron (the 6-7' fragment; Fig. $5 \mathrm{D}, \mathrm{G} /$ directs a weak dorsal-specific expression pattern. Using both deletion mutagenesis and point mutagenesis, we have resolved the activation function from the repression function of the central fragment and have mapped these functions to a small region of DNA (Figs. 8 and 9).

Although the multiple activators and repressors within the intron might seem to be a redundancy, these elements appear to work together to fine-tune the level and pattern of $d p p$ expression. An example of how this might work comes from comparing the expression patterns of the $6-7^{\prime}, 4-7^{\prime}$, and $2-7^{\prime}$ constructs (Fig. 5). The 6-7' fragment contains an enhancer and a ventral-spe-

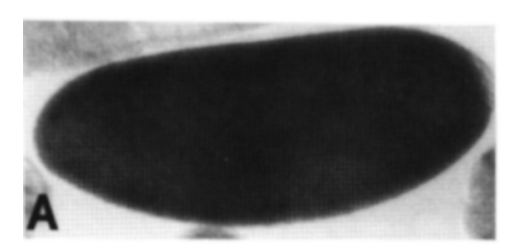

Figure 8. Dissection of the 3-5' region identifies a general enhancer element and a minimal ventral repressor region. lac $Z$ mRNA distributions are revealed by in situ hybridization with digoxigenin-labeled probes. Embryos, which are oriented as in Fig. 1, are viewed in sagittal section. The $4-5^{r}-980 / d p p$ construct directs uniform expression in the blastoderm embryo (A). The 8-8'-980/dpp/lacZ construct drives a dorsal-to-ventral graded expression pattern of the reporter gene $(B)$. The results are summarized in $C$. Solid ovals indicate $\mathrm{S} d \mathrm{l}$-binding sites; open ovals indicate $\mathrm{M} d \mathrm{l}$ binding sites (see text for definition of $S$ and $M$ sites).

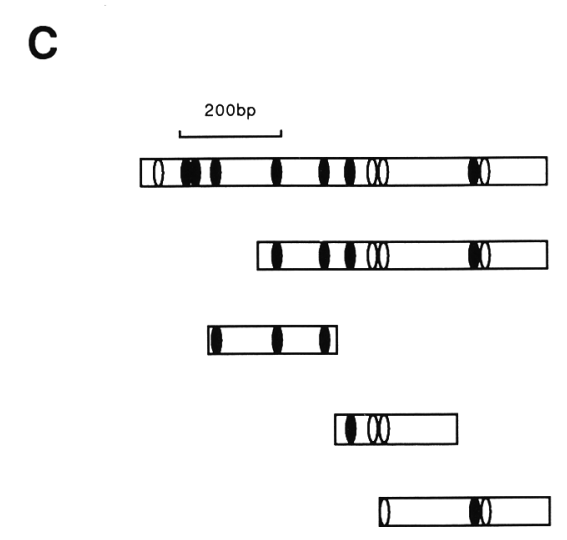

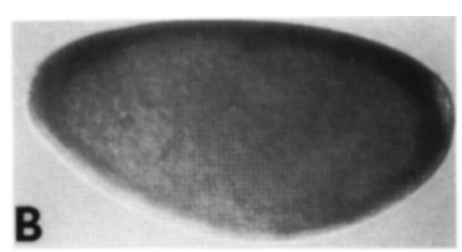

\begin{tabular}{|c|c|c|c|}
\hline \multirow{2}{*}{$\begin{array}{c}\text { DNA } \\
\text { Fragment }\end{array}$} & Early & & \multirow[t]{2}{*}{ Panels } \\
\hline & Dorsal & Ventral & \\
\hline $3-5^{\circ}$ & +++ & - & $\begin{array}{c}\text { Figure } \\
5 \mathrm{E}\end{array}$ \\
\hline $4-5^{\circ}$ & +++ & +++ & $8 \mathrm{~A}$ \\
\hline $8-8^{\circ}$ & ++ & + & $8 \mathrm{~B}$ \\
\hline $9-9^{\prime}$ & - & $\cdot$ & - \\
\hline $5-5^{\prime}$ & + & - & - \\
\hline
\end{tabular}


A

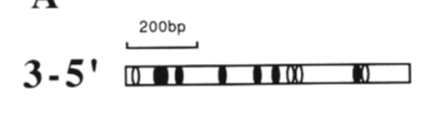

D

$3-5$ 'M एखि

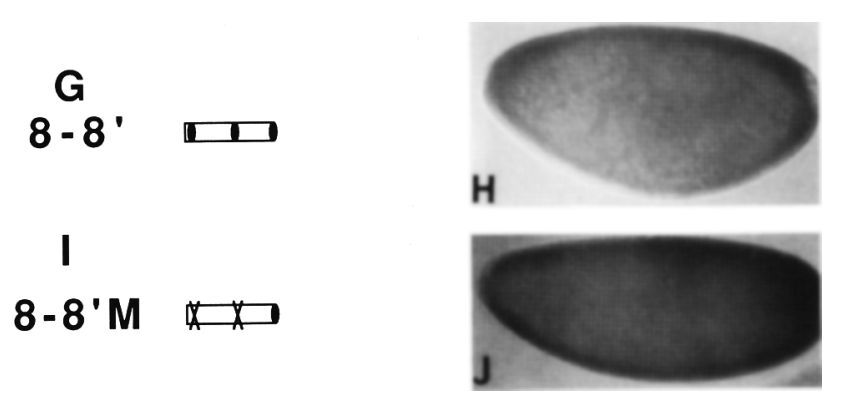

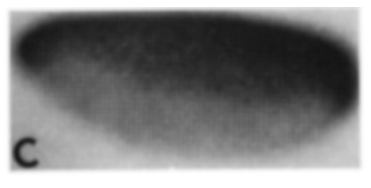

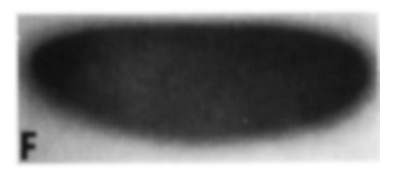

Figure 9. Ventral repression of $d p p$ is directly mediated by $d l$ binding sites. IacZ mRNA distributions are revealed by in situ hybridization with digoxigenin-labeled probes. Embryos, which are oriented as in Fig. 1, are viewed in sagittal section $(B, E, H, J)$ or on the surface $(C, F)$. All fragments are linked to $-980 / d p p$. The $3-5$ ' region with wild-type S3 and S4 $d I$ sites $(A)$ directs a dorsalspecific expression pattern $(B)$. The surface view $(C)$ shows that the expression domain has a discrete boundary. The 3-5' region with mutant S3 and S4 $d 1$ sites $(D)$ gives rise to a dorsal-to-ventral graded expression pattern of the reporter gene $(E)$. The surface view (F) shows no discrete boundary. The 8-8' region with wild-type S3 and S4 $d l$ sites $(G)$ has a dorsal-to-ventral graded expression pattern $(H)$. The 8-8' region with mutant S3 and S4 $d 1$ sites $\langle I|$ exhibits near uniform expression along the dorsal-ventral axis $(\eta)$. The embryo in $H$ was developed for half as long as the other embryos displayed, thus accounting for the weaker staining in $H$ relative to $J .(A, D, G, I)$ Solid ovals indicate $\mathrm{S} d l$-binding sites; open ovals indicate $\mathrm{M}$ $d l$-binding sites; $\times$ indicates mutated $d l$-binding site.

cific silencer, which together generate a low level of dorsal-specific expression. Addition of another enhancer element, as in the 4-7' fragment, results in near wild-type activity levels of expression dorsally. In the presence of two enhancers, however, the single ventral repressor element is no longer sufficient to completely repress transcription ventrally. Addition of another ventral repressor element, as in the $2-7^{\prime}$ fragment, then serves to completely restore ventral repression.

As is the case with zen (Ip et al. 1991), the ventral repression of $d p p$ involves the direct binding of the $d l$ transcription factor to the ventral repressor elements. Unlike zen, however, which contains a few $d l$-binding sites of high affinity, dpp appears to contain a large number of sites of relatively low affinity. This was somewhat surprising because the initial domains of expression for both genes are nearly identical (Ray et al. 1991), suggesting that the threshold level of $d l$ morphogen necessary to repress $d p p$ expression is very similar to the level required to repress zen expression. One might therefore expect the two genes to contain binding sites of similar affinity. There are various possible explanations for this unexpected result. For example, $d l$ could bind cooperatively to the many weak sites in $d p p$, thereby resulting in occupancy of these sites at a lower than expected concentration of the factor. Our data, however, would seem to argue against this possibility. In Figure 7D (lanes 1-4), the binding of a zen VRE fragment containing a single high-affinity $d l$ site is compared with the binding of a $d p p$ second intron fragment containing five low-affinity sites. Despite the multiple sites in the $d p p$ fragment and the resultant possibility for cooperative binding, the $d p p$ fragment binds $d l$ protein far less well than the zen frag- ment. On the other hand, it is possible that cooperativity would only be manifested when the DNA is assembled into chromatin, as has been observed for the binding of the yeast transcription factor GAL4 (Taylor et al. 1991).

Alternatively, perhaps other proteins can associate with the $d l$ protein in vivo, modifying its affinity for certain sites. Rel family proteins such as $d l$ are believed to bind DNA as dimers, and certain family members are known to associate with one another to create heterodimeric proteins (Rushlow and Warrior 1992). Because the different family members have slightly different specificities, the formation of heterodimers is thought to be a way of creating additional specificities from a limited number of protomers. Although $d l$ protein is believed to self-associate (Govind et al. 1992), perhaps it can also form heterodimers with an unidentified rel family protein.

In accord with the results presented here pertaining to dl-mediated repression, previous studies indicate that the breadth of a dl-mediated activation domain is likewise not a simple function of the $\mathrm{dl}$-binding affinity of the target gene (Pan and Courey 1992; Jiang et al. 1992). For example, multimerized high-affinity $\mathrm{dl}$-binding sites were found to direct the ventral activation of the hsp 70 core promoter element. This domain of activation, however, was not significantly wider than that observed for the twist ventral activator region, which contains multiple low-affinity $d l$-binding sites.

How does $d l$ bound to ventral repressor elements prevent the function of linked enhancers? Although we do not yet have the answer to this question, it is clear that VREs utilize a long-range mechanism that is distinct from the short-range mechanism that has been proposed 
to play an important role in generating the expression domains of some gap and pair-rule genes. For example, $K r$ is believed to repress even-skipped $(e v e)$ in the region between eve stripes 2 and 3 by binding to sites overlapping the binding sites for critical activators of eve, thereby blocking activation (Small et al. 1991). Our results show that general activators in the $d p p$ second intron are fairly closely linked to $d l$-dependent repressor elements; thus, this type of short-range repression could be partly responsible for the ventral silencing of $d p p$. We know that this short-range mechanism, however, is not the sole mechanism of $d l$-mediated repression, because VREs can work over long distances to block the activity of activators that must be binding to nonoverlapping sites (e.g., see Fig. 4).

In addition, we also know that VREs work by a mechanism that is distinct from that defined for the suppressor of Hairy-wing binding region in the gypsy transposon (Geyer and Corces 1992) or the specialized chromatin structures associated with polytene chromosome puffs (Kellum and Schedl 1991). These repressor elements only work when they are placed between an enhancer and a core promoter element. It is therefore suspected that they serve to block the tracking of some factor along the DNA from the enhancer to the core promoter. Both our results (Fig. 4) and the studies on zen (Doyle et al. 1989; Ip et al. 1991; Jiang et al. 1992) clearly show that dIdependent VREs prevent activation even when they are positioned upstream of an enhancer.

Numerous studies point to the idea that regulatory proteins can influence rates of transcription via direct interactions with the general transcriptional machinery (Ptashne and Gann 1990). Conceivably, dl bound to a VRE could form a nonproductive complex with the general machinery, thereby preventing activators from functioning; however, $d l$ can also serve as an activator, therefore, we need to propose an additional mechanism that allows $d l$ to activate some promoters and repress others. As noted above, previous studies have shown that a multimerized $d l$-binding site from the zen VRE inserted upstream of a core promoter element serves as a $d l$-dependent ventral activator element (Pan and Courey 1992; Jiang et al. 1992). This result indicates that by itself, $d l$ has an activation function and that repression may require an interaction between $\mathrm{dl}$ and an unidentified corepressor. Presumably, this corepressor is brought to the DNA through a sequence-specific protein-DNA interaction involving some site separate from the $d I$-binding sites. It could be the complex of this DNA-bound corepressor with $d l$ that interacts with the general machinery to antagonize activator function. The interaction between the putative corepressor and $d I$ protein could also result in cooperative DNA binding, effectively converting a weak $d l$-binding site into a strong site. If so, this could explain the ability of $d l$-binding sites of different affinity to mediate the same domain of ventral repression.

Additional evidence for the importance of the position of $d l$-binding sites with respect to binding sites for other proteins comes from an analysis of the expression pat- tern directed by the 4-5' fragment of the $d p p$ second intron (Fig. 8A,C). This fragment contains multiple dlbinding sites, and yet it mediates an expression pattern that is uniform along the dorsal-ventral axis, whereas the 3-5' fragment (which contains an additional $240 \mathrm{bp}$ of intronic sequences; Fig. 5E,G) directs a dorsal-specific expression pattern. The extra sequences in the 3-5' fragment may bind a protein that serves a corepressor function. An analysis of the $1 a c Z$-coding sequences present in all our P-element constructs also suggests that the context of $d l$-binding sites is important. The lacZ-coding region contains several matches for the consensus $d l$ binding site (data not shown). Yet these sites mediate neither transcriptional activation nor transcriptional repression. Apparently, these binding sites are not properly situated with respect to binding sites for other proteins and/or with respect to one another.

These studies have identified a 270-bp fragment of the second intron (the $8-8^{\prime}$ fragment) that is likely to contain binding sites for proteins that activate $d p p$ as well as binding sites for the putative corepressor that converts $d l$ from an activator into a repressor. Neither the activators nor the putative corepressors regulating $d p p$ expression have been identified by genetic screens. This could be attributable to the redundancy of these factors or to pleiotropic effects of mutations in the genes encoding them. Identification of these factors may be achieved more readily by biochemical means. These studies provide a good starting point for that analysis because they have identified potential target regions for these factors in the DNA.

\section{Materials and methods}

\section{P-element constructs}

To make the P-element constructs with different amounts of 5 '-flanking sequences, the $d p p$ EcoRI-SmaI fragment was first cloned into pBluescript (Stratagene) between the EcoRI and SmaI sites. The resulting plasmid was linearized by cutting with EcoRI, deleted to a variable extent with exonuclease III and mung bean nuclease, and then restricted with NotI. DNA fragments with different $5^{\prime}$ ends were resolved on an agarose gel and cloned into pBluescript linearized with $S m a \mathrm{I}$ and NotI. The $5^{\prime}$ ends of these constructs were determined by DNA sequencing. The constructs with $980,582,98,46$, and 22 bp of $d p p 5^{\prime}$-flanking sequences were cut with $N c o l$, blunt-ended with $\mathrm{T}_{4}$ DNA polymerase, and digested with $E c o R I$. The resulting $d p p$ fragments were cloned into the pCaSpeR-AUG- $\beta$ gal P-element vector (Thummel et al. 1988) that had been cut with EcoRI and $B a m H I$ (the BamHI site was blunt-ended with $\mathrm{T}_{4}$ DNA polymerasel.

The fragment containing the full-length second intron plus a small amount of sequence from the flanking exons extends from +1047 to +3031 relative to the transcriptional start site. Different $d p p$ second-intron fragments were amplified by PCR with primers from the positions shown in Figure 5G. These primers each contain 28-30 nucleotides of $d p p$ sequence, as well as $E c o$ RI sites at their $5^{\prime}$ ends. The $d p p$ homologous sequences in each of the primers have their 5' ends at the following position relative to the transcriptional start site: $1,+1129 ; 2,+1369 ; 3$, $+1609 ; 4,+1849 ; 5,+2089 ; 6,+2329 ; 7,+2569 ; 8,+1741 ; 9$, $+1990 ; 1^{\prime},+1509 ; 2^{\prime}{ }^{\prime}+1668 ; 3^{\prime},+1908 ; 4^{\prime},+2148 ; 5^{\prime}$, 
$+2400 ; 6^{\prime},+2628 ; 7^{\prime},+2988 ; 8^{\prime},+2005 ;$ and $9^{\prime},+1989$. The amplified DNA fragments were treated with EcoRI and cloned into the EcoRI site of the P-element vectors with different $5^{\prime}$ $d p p$ flanking sequences.

Oligonucleotide-directed mutagenesis was carried out by standard procedures (Ausubel et al. 1991). Mutants were identified by the additional restriction site introduced by the mutagenesis. The ability of these sites to form complex with $d l$ protein was abolished as determined by mobility retardation assay. In the mutant $8-8^{\prime}$ and $3-5^{\prime}$ fragments, the S3 site was mutated from CGGATTTTCC to CATCTGCAG and the S4 site was mutated from CGGAAATTCC to CTAAGCTTCA.

To make the $d p p$ second intron- $h s p 70 / l a c Z$ P-element construct, a Notl fragment containing the entire second intron was cloned into the NotI site of a P-element vector that contains the hsp70 core promoter element driving expression of lacZ [provided by G. Liaw and J. Lengyel (Pan et al. 1991)]. The $d p p$ intron-Kr-hsp $70 / 1 a c Z$ construct was made by inserting the NotI fragment into the NotI site of a $K r-h s p 70 / l a c Z$ P-element construct provided by T. Ip and M. Levine (Ip et al. 1991).

Orientations of the inserts were determined by PCR with one primer in the intron region and a second primer in the $5^{\prime} d p p$ flanking region. The orientation of the intron inserts was not observed to have any effect on the observed expression patterns. For the data shown in this paper, most of these inserts were determined to be in the + orientation (i.e., the orientation relative to the transcriptional start site was the same as in the intact gene), with the following exceptions: $5-5^{\prime}-980 / d p p$ and $3-5^{\prime}$ (S3, S4 mutation) $-980 / d p p$ were both determined to be in the - orientation; for $1-7^{\prime}-980 / d p p, 1-7^{\prime}-582 / d p p$, $1-7^{\prime}-346 / d p p, 1-7^{\prime}-98 / d p p, 1-7^{\prime}-46 / d p p, 1-7^{\prime}-22 / d p p$, intron-hsp70/lacZ, and intron-Kr-hsp70/lacZ, the orientations were not determined.

\section{Analysis of expression by whole-mount in situ hybridization}

DNA or RNA probes for the $d p p$ - and lacZ-coding regions were labeled with digoxigenin according to the protocol supplied by Boehringer Mannheim with their kit (cat. 1093-657) or as described by Jiang et al. (1991). Embryos from multiple independent transformed lines of each construct were collected and fixed. Hybridization conditions were as described by Tautz and Pfeifle (1989) or Jiang et al. (1991). The estimates of the relative strengths of dorsal and ventral expression, as indicated by the numbers of pluses in Figures 5G and 8C, were obtained by staining multiple lines of each construct for equivalent amounts of time in side-by-side reactions.

\section{Expression of $\mathrm{dl}$ protein and DNA-binding assays}

Electrophoretic mobility-shift assays (EMSA) used a truncated version of the $d l$ protein produced in Escherichia coli as described by Pan et al. (1991). DNase I footprinting assays used full-length $d l$ protein expressed using a baculovirus expression vector and standard procedures (Ausubel et al. 1991). The protein was isolated from extracts of the nuclei of baculovirusinfected Sf 9 cells and purified to homogeneity by two rounds of DNA affinity chromatography (Kadonaga and Tjian 1986) using a column made with an oligonucleotide containing a high-affinity $d l$-binding site. The two strands of the oligonucleotide were GATCGATTGGGTTTCTCCCAGTTA and GATCTAACTGGGAGAAACCCAATC.

DNase I footprinting reactions were carried out as described previously (Heberlein et al. 1985). EMSA was carried out as described by Ip et al. (1991). The consensus-binding site EMSA probe was identical to the oligonucleotide used in the DNA affinity purification of baculovinus $d l$ protein.

\section{Drosophila strains}

P-element constructs were injected into $w^{1118}$ or $r y^{42}$ flies. To examine the dorsal dependence of the dorsal-specific expression of the reporter gene, $w^{1118}$ flies bearing a P-element insertion were crossed to $T o l^{10 b}$ females or homozygous $d l^{1}$ females.

\section{Acknowledgments}

We thank Rick Padgett for communicating his unpublished results. We gratefully acknowledge Gwo-Jen Liaw for his technical advice and assistance; and Tony Ip, Jin Jiang, and Mike Levine for their generosity with research materials. We also thank Mike Carey, Steve Crews, Jay Gralla, and Judy Lengyel for their insightful comments on the manuscript. This work was supported by grants to A.J.C. from the National Institutes of Health, the Searle Scholars Program, and the March of Dimes. J.H. is a recipient of a Sigma Xi Grant-In-Aid of Research from the National Academy of Sciences.

The publication costs of this article were defrayed in part by payment of page charges. This article must therefore be hereby marked "advertisement" in accordance with 18 USC section 1734 solely to indicate this fact.

\section{References}

Anderson, K.V. 1989. Drosophila: The maternal contribution. In Gametogenesis and the early embryo (ed. D.M. Glover and B.D. Hames), pp. 1-37. IRL Press, Oxford, England.

Ausubel, F.M., R. Brent, R.E. Kingston, D.D. Moore, J.G. Seidman, J.A. Smith, and K. Struhl. 1991. Current protocols in molecular biology. John Wiley, New York.

Doyle, H.J., R. Kraut, and M. Levine. 1989. Spatial regulation of Zerknüllt: A dorsal ventral patterning gene in Drosophila. Genes \& Dev. 3: 1518-1533.

Ferguson, E.L. and K.V. Anderson. 1992. decapentaplegic acts as a morphogen to organize dorsal-ventral pattern in the Drosophila embryo. Cell 71: 451-461.

Geyer, P.K. and V.G. Corces. 1992. DNA position-specific repression of transcription by a Drosophila zinc finger protein. Genes \& Dev. 6: 1865-1873.

Govind, S., A.M. Whalen, and R. Steward. 1992. In vivo selfassociation of the Drosophila rel-protein dorsal. Proc. Natl. Acad. Sci. 89: 7861-7865.

Heberlein, U., B. England, and R. Tjian. 1985. Characterization of Drosophila transcription factors that activate the tandem promoters of the alcohol dehydrogenase gene. Cell 41: 965977.

Hoch, M., C. Schroder, E. Seifert, and H. Jackle. 1990. Cis-acting control elements for Krüppel expression in the Drosophila embryo. EMBO I. 9: 2587-2595.

Ip, Y.T., R. Kraut, M. Levine, and C.A. Rushlow. 1991. The dorsal morphogen is a sequence-specific DNA-binding protein that interacts with a long-range repression element in Drosophila. Cell 64: 439-446.

Ip, Y.T., R.E. Park, D. Kosman, K. Yazdanbakhsh, and M. Levine. 1992a. dorsal-twist interactions establish snail expression in the presumptive mesoderm of the Drosophila embryo. Genes \& Dev 6: 1518-1530.

Ip, Y.T., R.E. Park, D. Kosman, E. Bier, and M. Levine. 1992b. The dorsal gradient morphogen regulates stripes of rhomboid expression in the presumptive neuroectoderm of the 
Drosophila embryo. Genes \& Dev. 6: 1728-1739.

Irish, V.F. and W.M. Gelbart. 1987. The decapentaplegic gene is required for dorsal-ventral patterning of the Drosophila embryo. Genes \& Dev. 1: 868-879.

Jiang, J., D. Kosman, Y.T. Ip, and M. Levine. 1991. The dorsal morphogen gradient regulates the mesoderm determinant twist in early Drosophila embryos. Genes \& Dev. 5: 18811891.

Jiang, J., C.A. Rushlow, Q. Zhou, S. Small, and M. Levine. 1992. Individual dorsal morphogen binding sites mediate activation and repression in the Drosophila embryo. EMBO $J$. 11: $3147-3154$.

Kadonaga, J.T. and R. Tjian. 1986. Affinity purification of sequence-specific DNA binding proteins. Proc. Natl. Acad. Sci. 83: 5889-5893.

Kasai, Y., J.R. Nambu, P.M. Lieberman, and S.T. Crews. 1992. Dorsal-ventral patterning in Drosophila-DNA binding of snail protein to the single-minded gene. Proc. Natl. Acad. Sci. 89: 3414-3418.

Kellum, R. and P. Schedl. 1991. A position-effect assay for boundaries of higher order chromosomal domains. Cell 64: 941-950.

Padgett, R.W., J.R. St. Johnston, and W.M. Gelbart. 1987. A transcript from a Drosophila pattern gene predicts a protein homologous to the transforming growth factor-beta family. Nature 325: 81-84.

Pan, D. and A.J. Courey. 1992. The same dorsal binding site mediates both activation and repression in a context-dependent manner. EMBO I. 11: 1837-1842.

Pan, D.J., J.D. Huang, and A.J. Courey. 1991. Functional analysis of the Drosophila twist promoter reveals a dorsal-binding ventral activator region. Genes \& Dev. 5: 1892-1901.

Ptashne, M. and A.A. Gann. 1990. Activators and targets. Nature 346: 329-331.

Ray, R.P., K. Arora, C. Nüsslein-Volhard, and W.M. Gelbart. 1991. The control of cell fate along the dorsal ventral axis of the Drosophila embryo. Development 113: 35-54.

Roth, S., D. Stein, and C. Nüsslein-Volhard. 1989. A gradient of nuclear localization of the dorsal protein determines dorsoventral pattern in the Drosophila embryo. Cell 59: 11891202.

Rushlow, C. and R. Warrior. 1992. The rel family of proteins. BioEssays 14: 89-95.

Rushlow, C., M. Frasch, H. Doyle, and M. Levine. 1987. Maternal regulation of zerknüllt: A homoeobox gene controlling differentiation of dorsal tissues in Drosophila. Nature 330: $583-586$.

Rushlow, C.A., K. Han, J.L. Manley, and M. Levine. 1989. The graded distribution of the dorsal morphogen is initiated by selective nuclear transport in Drosophila. Cell 59: 11651177 .

St. Johnston, R.D. and W.M. Gelbart. 1987. Decapentaplegic transcripts are localized along the dorsal-ventral axis of the Drosophila embryo. EMBO I. 6: 2785-2791.

St. Johnston, R.D., F.M. Hoffmann, R.K. Blackman, D. Segal, R. Grimaila, R.W. Padgett, H.A. Irish, and W.M. Gelbart. 1990. Molecular organization of the decapentaplegic gene in Drosophila melanogaster. Genes \& Dev. 4: 1114-1127.

Shimell, M.J., E.L. Ferguson, S.R. Childs, and M.B. O'Connor. 1991. The Drosophila dorsal-ventral patterning gene tolloid is related to human bone morphogenetic protein-1. Cell 67: 469-481.

Small, S., R. Kraut, T. Hoey, R. Warrior, and M. Levine. 1991. Transcriptional regulation of a pair-rule stripe in Drosophila. Genes \& Dev. 5: 827-839.

Steward, R. 1989. Relocalization of the dorsal protein from the cytoplasm to the nucleus correlates with its function. Cell 59: 1179-1188.

Tautz, D. and C. Pfeifle. 1989. A non-radioactive in situ hybridization method for the localization of specific RNAs in Drosophila embryos reveals translational control of the segmentation gene hunchback. Chromosoma 98: 81-85.

Taylor, I.C.A., J.L. Workman, T.J. Schuetz, and R.E. Kingston. 1991. Facilitated binding of GAL4 and heat shock factor to nucleosomal templates-Differential function of DNAbinding domains. Genes \& Dev. 5: 1285-1298.

Thisse, B., C. Stoetzel, M. El Messal, and F. Perrin-Schmitt. 1987. Genes of the Drosophila maternal dorsal group control the specific expression of the zygotic gene twist in presumptive mesodermal cells. Genes \& Dev. 1: 709-715.

Thisse, C., F. Perrin-Schmitt, C. Stoetzel, and B. Thisse. 1991. Sequence-specific transactivation of the Drosophila twist gene by the dorsal gene product. Cell 65: 1191-1201.

Thummel, C.S., A.M. Boulet, and H.D. Lipshitz. 1988. Vectors for Drosophila P-element-mediated transformation and tissue culture transfection. Gene 74: 445-456. 


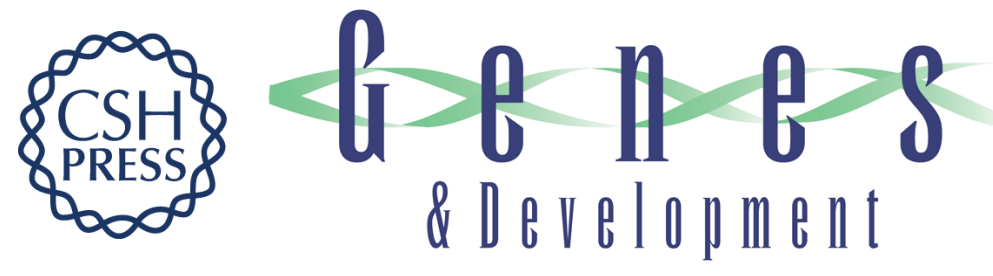

\section{The interplay between multiple enhancer and silencer elements defines the pattern of decapentaplegic expression.}

J D Huang, D H Schwyter, J M Shirokawa, et al.

Genes Dev. 1993, 7:

Access the most recent version at doi:10.1101/gad.7.4.694

References This article cites 34 articles, 15 of which can be accessed free at:

http://genesdev.cshlp.org/content/7/4/694.full.html\#ref-list-1

License

Email Alerting

Service

Receive free email alerts when new articles cite this article - sign up in the box at the top right corner of the article or click here.

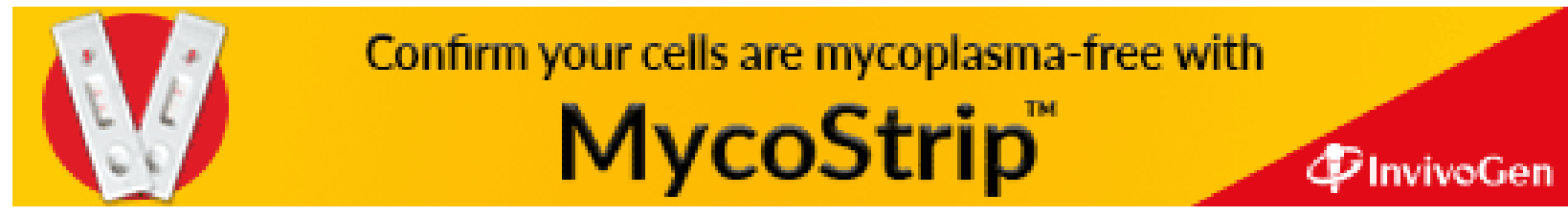

